\title{
Aspectos epistemológicos a luz da teoria antropológica do didático subjacentes ao tema transformação linear
}

Epistemological aspects in the light of the anthropological theory of the didactics underlying the linear transformation theme

\author{
Fernando Cardoso de Matos ${ }^{1}$ \\ José Carlos de Souza Pereira² \\ José Messildo Viana Nunes ${ }^{3}$ \\ Renato Borges Guerra ${ }^{4}$
}

\section{Resumo}

Nosso propósito neste artigo foi revelar os aspectos epistemológicos a luz da Teoria Antropológica do Didático estão subjacentes ao tema transformação linear, no livro didático adotado para o ensino de Álgebra Linear no Instituto Federal do Pará, revelando indícios do modelo epistemológico dominante, analisando dois tipos de tarefas. A análise do livro didático é um referencial para a elaboração de conjecturas a respeito do tipo de ensino que está sendo desenvolvido de um determinado saber, em uma determinada instituição. A Teoria nos ajudou a discutir Organização Matemática e Didática do livro, em termos do gênero de tarefas e tipos de tarefas, técnicas e justificações da razão de ser do objeto matemático transformação linear. A razão de ser para se ensinar esse objeto se dá pela aplicação direta da definição, pois os alunos devem se perguntar ao lerem as propostas das tarefas enunciadas pelos autores, para que e porque se estudar tal temática? Algumas tarefas são resolvidas com os conteúdos vistos no ensino médio, como função como tecnologia, não deixando claro ao leitor que é uma transformação de um espaço vetorial em outro. Concluímos que nos tipos de tarefa discutidos foi possível dar indícios do modelo epistemológico dominante, o qual consideramos a utilização direta da definição de transformação linear.

Palavras chave: Teoria Antropológica do Didático; Transformações Lineares; Organização Praxeológica.

\footnotetext{
${ }^{1}$ Universidade Federal do Pará | matos2001@gmail.com

${ }^{2}$ Universidade Federal do Pará | jsouzaper@gmail.com

${ }^{3}$ Universidade Federal do Pará | messildo@ufpa.br

${ }^{4}$ Universidade Federal do Pará | rguerra@ufpa.br
} 


\section{Abstract}

Our purpose in this article was to reveal the epistemological aspects in the light of the anthropological theory of the Didactics are underlying the theme linear transformation in the textbook adopted for the teaching of Linear Algebra at the Federal Institute of Pará, revealing evidence of the dominant epistemological model, analyzing two types of tasks. The analysis of the didactic book is a reference for the elaboration of conjectures regarding the type of teaching that is being developed of a certain knowledge, in a certain institution. The Theory helped us to discuss Mathematical Organization and Didactics of the book in terms of the gender of tasks and task types, techniques and justifications of the rationale of mathematical object linear transformation. The reason for being to teach this object is given by the direct application of the definition, because the students should ask themselves when reading the proposals of the tasks enunciated by the authors, so that and why to study this subject? Some tasks are solved with the contents seen in high school, as a function as technology, not making it clear to the reader that it is a transformation from one vector space to another. We conclude that in the types of tasks discussed it was possible to give evidence of the dominant epistemological model, which we consider the direct use of the definition of linear transformation.

Keywords: Anthropological Theory of Didactics; Linear Transformations; Praxeological Organization.

\section{Introdução}

Nos diversos sistemas de ensino e em seus sistemas didáticos ${ }^{5}$ o ensino da Álgebra se configura como um processo dificultoso para a maioria dos estudantes. A Álgebra é apresentada nos livros didáticos a partir do sétimo ano do ensino fundamental, porém quase sem qualquer articulação com que o aluno já tem de conhecimento. $\bigcirc$ mesmo acontecendo no ensino superior, que pouco se articula com a Álgebra do ensino básico.

Telles (2004) relata que ao longo dos séculos, os matemáticos foram lentamente aprendendo a substituir as palavras por letras e por sinais: $=,+,-, x_{1} \div$. Esse processo de simbolização permitiu surgir às noções da Álgebra, que transgrediu a Geometria Euclidiana. A palavra álgebra tem sua etimologia, que é uma variante latina da palavra árabe al-jabr, usada no título de um livro, Hisab al-jabr w'al-muqabalah, escrito em Bagdá por volta do ano 825 .

Ao consultarmos os livros didáticos de Álgebra Linear, presentes na ementa do curso de Licenciatura em Matemática do Instituto Federal do Pará (IFPA), na qual atuamos como professores de Álgebra Linear (AL), percebe-se que esta disciplina tem seu lugar reservado, pois é de extrema importância para um professor de matemática, além de servir de base para diversas outras disciplinas, porém a abstração axiomática decorrente do formalismo, que seus conteúdos possuem é notável, talvez isso seja um dos obstáculos didáticos que permeia o sistema didático dessa disciplina.

Em boa parte dos livros de AL adotados pelas instituições de ensino superior os sistemas de tarefas não começam com as tarefas mais simples e rotineiras, que Gascón

\footnotetext{
${ }^{5}$ Sistema constituído pelo professor, alunos e um saber (CHEVALLARD, 1994, p. 175).
} 
(2001) intitula de genéricas até as mais complexas (problemáticas) ou mais especificas. Segundo Andrade e Guerra (2014), nesse processo, as tarefas problemáticas são tornadas rotineiras, no sentido de poderem ser realizadas de forma simples, rápida e seguras, por meio de elaboradas maneiras de fazer, ou técnicas, eficientes, inteligíveis e justificados.

Para realizarmos esse estudo nos baseamos na Teoria Antropológica do Didático (TAD) (CHEVALLARD, 1999), devido seu potencial analítico, que é capaz de revelar o caminho percorrido pelos saberes, inclusive os escolares, além de viabilizar um olhar detalhado de suas especificidades, mostrando-se essencial para fornecer respostas às questões problemáticas dos sistemas de didáticos.

A TAD é aqui a teoria que visa à discussão da Organização Matemática (OM) do livro didático, em termos do gênero de tarefas e tipo de tarefas, técnicas e justificações da razão de ser do objeto matemático Transformação Linear $(\mathrm{TL})$, para dar indícios do modelo epistemológico dominante ${ }^{6}$ na instituição IFPA. Tal objeto é visto no curso superior de Licenciatura em Matemática e tem demonstrado ser um obstáculo para os alunos, conforme postulam á que Dorier (1995), Dorier (1997) e Dorier et al. (1994) revelam que o formalismo causa dificuldade na aprendizagem desta disciplina.

Segundo Boch e Gascón (2010), a forma da OM escolar no ensino, apoia-se de certa maneira em um Modelo Epistemológico (ME), que está relacionado ao que se refere a ensinar e aprender matemática e uma instituição (I). O ME nos permite compreender a OM dos livros didáticos e sua influência na prática do professor nos permitindo fornecer indícios de um modelo epistemológico dominante na I livro.

Nessa perspectiva temos como objetivo revelar aspectos epistemológicos subjacentes ao tema transformação linear, no livro Alfredo Steinbruch e Paulo Winterle (1987), intitulado Álgebra Linear, a luz da TAD, adotado como referência na instituição IFPA, revelando indícios do Modelo Epistemológico Dominante (MED), analisando dois tipos de tarefas.

A TAD como ferramenta teórico-metodológica nos possibilita explicitar o gênero, a técnica, a tecnologia e a teoria, verificando como as transformações lineares se apresentam na Organização Matemática (OM) destinada a seu ensino, dando indícios do MED na instituição IFPA, já que o livro didático é o livro texto adotado pelos professores do IFPA para ministrar esta disciplina, tornando-se assim um indicativo de relevância da TAD num curso de formação inicial de futuros professores de matemática.

\section{O livro didático}

O livro didático legitima-se nas práticas sociais validadas e é um importante recurso utilizado pelos professores para elaboração de suas aulas, portanto desempenha um papel de referência na atividade do professor do ensino superior, pois o professor é responsável por recriar um saber?, isto é, em fazer o que Yves Chevallard chama de transposição didática interna, quando adéqua um determinado conteúdo para estruturar suas aulas, ou seja, realiza a transformação do saber a ensinar em saber ensinado, na sala de aula.

\footnotetext{
${ }^{6}$ É a forma predominante de descrever uma organização matemática em uma dada instituição.

${ }^{7}$ Da a ideia de um modelo, mas na realidade é um termo ainda indefinido, pois o que se tem é a relação com o saber.
} 
A prática de criação dos saberes pelos professores está relacionada diretamente as obras estudadas por esses ao longo de sua vida, nesse sentido a análise dos livros didáticos é um referencial para a elaboração de conjecturas a respeito do tipo de ensino que está sendo desenvolvido de um determinado saber, em uma determinada instituição.

Segundo Menssouri (1994, p.46), para sabermos como um saber (um modelo) vive em uma instituição particular é necessário efetuar a análise dos livros didáticos, pois: "... os livros didáticos constituem uma realização efetiva e objetiva do ensino realizado em classe. Realização que é submetida ao olhar e ao julgamento público, e representativo da realidade da classe."

Para Carvalho e Lima (2010), o livro é portador de escolhas sobre o saber a ser estudado, os métodos adotados para que os alunos consigam aprendê-lo mais eficazmente e a organização curricular ao longo dos anos de escolaridade. Ainda segundo esses pesquisadores, nesse diálogo está entrelaçada a relação que envolve os autores do livro didático, o professor, o aluno e a Matemática. Essas ideias traduzem-se, conforme Figura 1:

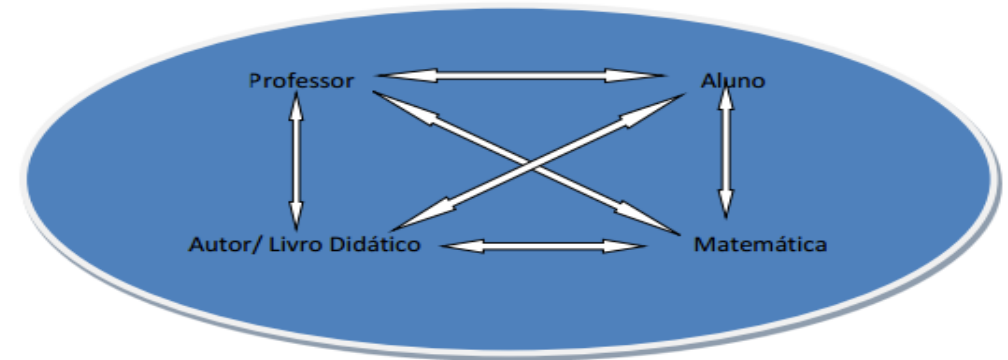

Figura 1 - O livro didático na sala de aula. Fonte: (CARVALHO; LIMA, 2010, p. 15).

Ao professor cabe conduzir o processo de ensino e que, principalmente, no nível superior o livro é uma das principais fontes de consulta, auxiliando na gestão do saber, sendo que de certa forma há um diálogo entre os autores com seus leitores, o professor e o aluno, como uma teia, conforme Figura 1. Ao nosso ver o livro é um recurso, mas não deve ocupar o papel dominante no processo de ensino, comprometendo a autonomia do professor.

\section{A Teoria Antropológica do Didático (TAD)}

Da mesma maneira que existe uma antropologia religiosa, cujo objeto de estudo é a religião, Chevallard (1992) propôs elaborar uma antropologia didática, cujo objeto de estudo seria a didática, a fim de estudar, por exemplo, fenômenos acerca das praxeologias do professor de matemática diante de um objeto matemático, tal como as TL.

A TAD de Yves Chevallard, cujo objeto de investigação consiste na análise da atividade matemática, como por exemplo, a escolar, e suas relações humanas enquadradas em determinadas instituições sociais ${ }^{8}$.

\footnotetext{
${ }^{8}$ Para Chevallard, Bosch e Gascón (2001) nesse sentido, tanto o conhecimento como as atividades matemáticas são construções sociais que se realizam em instituições, em comunidades, seguindo determinados contratos
} 
Chevallard (1999) coloca a TAD como uma teoria, que possui um método de análise para organização matemática existente no interior de uma determinada instituição de ensino. No artigo "Concepts fondamentaux de la Didactique: perspectives apportées par um approche anthropologique. In: Recherchesen Didactique dês Mathématiques" (CHEVALLARD, 1992) [Conceitos fundamentais de didática: perspectivas fornecidas por uma abordagem antropológica. In: Pesquisa em Educação Matemática] relata que a TAD representa uma base para a análise dos livros didáticos, além do potencial analítico em descrever o caminho percorrido pelos saberes escolares, fornecendo uma boa visão de suas especificidades.

Chevallard (1996) apresenta três elementos primitivos da TAD: os objetos O, as pessoas $X$ e as instituições I, ou seja, as I são o modo de fazer e de pensar. O objeto O tomará uma posição privilegiada em relação aos outros elementos, em virtude do mesmo ser o "material base" da construção teórica. O objeto passa a existir no momento em que for reconhecido como existente por pelo menos uma pessoa $X$ ou instituição $I$. A relação pessoal de $X$ com $O$, que será denotada por $R(X, O)$, e a relação institucional de I com $O$ por $R(I, O)$, e se dá no seio de I. Assim, estas relações são práticas sociais com o objeto.

Segundo Chevallard (1999), toda atividade humana regularmente realizada pode descrever-se com um modelo mínimo (práticas), que se resume aqui com a palavra praxeologia. A TAD o ensino da matemática em qualquer instituição / é descrito em termos de praxeologias de ensino, ou seja, a ação do sujeito. O saber matemático, enquanto forma particular do conhecimento é fruto da ação humana institucional, e é algo que se produz, se utiliza, se ensina ou, de uma forma geral, que transita nas instituições $I$.

A palavra praxeologia é formada por dois termos gregos, práxis e logos, que significam, respectivamente, prática e razão e remete ao fato de que, no interior de uma instituição I, a prática está sempre acompanhada de um discurso, ou seja, de um logos que a justifica, que Ihe dá razão, que se constroem em um processo dialético. Na raiz da noção de praxeologia, encontram-se as noções de tarefas e de tipos de tarefas.

Em uma organização praxeológica, identificamos: tarefas, técnicas, tecnologias e teorias. Na essência da noção de praxeologia se encontra as noções de tarefas e de tipos de tarefas, denotadas, respectivamente, por t e T. Quando uma tarefa t que faz parte de um tipo de tarefa $T$, dizemos que $t \in T$ (t pertence a $T$ ).

Em termos praxeológicos, podemos entender a Tarefa (t), que está sempre relacionada a um Tipo de tarefas (T), como toda ação singular, particular, específica de um fazer que se expressa por um verbo, como: arrumar a sala; organizar a gaveta; encontrar a fração reduzida; fatorar o polinômio; simplificar a expressão algébrica; encontrar a equação da reta tangente à curva no ponto $\mathrm{P}$; dividir um número por outro etc.

Já o Tipo de tarefas (T), é um conjunto de ações do mesmo tipo, ou seja, é uma classe de tarefas com características comuns, como: arrumar salas; organizar cômodas; simplificar expressões algébricas; encontrar equações de retas tangentes a uma curva em um dado ponto $P_{\text {; }}$ determinar $O$ quociente entre dois números dados etc., isto é, $T=\left\{t_{1}, t_{2}, t_{3}, t_{4}, \ldots \ldots \ldots t_{n}\right\} \ldots$

As noções de tarefa e tipos de tarefas, supõe objetos relativamente precisos e estão intimamente relacionadas a uma ideia mais ampla, o de gênero de tarefas, como por 
exemplo calcular, fatorar, transfomar, determinar, etc. Determina de certa forma o que fazer para solucionar determinados problemas e são compreendidas por estarem culturalmente instituidas.

A análise destas tarefas fornece um diagnóstico de porque há dificuldades de aprendizagem em relação ao objeto Transformação Linear, pois a Álgebra Linear é uma disciplina que possui objetos $O$ com alto grau de abstração.

Chevallard (1999) nos indica a necessidade dessas noções tornarem-se objetos de estudo ao destacar que :

Tarefas, tipo de tarefas e gênero de tarefas não são dados da natureza: são artefatos, obras, construções intitucionais, cujas reconstrução em tal instituição, por exemplo, em uma didática (CHEVALLARD, 1999, p. 3, tradução nossa).

A ideia de gênero de tarefas, que no decorrer dos anos de estudo esta noção esta sujeita ao processo de transacionalidade do objeto, visto que o gênero de tarefa Calcular pode significar efetuar uma expressão aritmética, ou efetuar uma expressão aritmética, determinar o número de anagramas de uma palavra dada. Assim sendo os gêneros de tarefas se enriquecem e aprimoram-se na sucessão dos anos letivos (SABO, 2001, p. 11).

A existência de uma técnica (denotada por $\tau$ ) supõe a existência subjacente de um discurso interpretativo e justificativo da técnica e de seu âmbito de aplicabilidade e validade, a tecnologia $\theta$ da técnica $\tau$. O discurso (tecnologia $\theta$ ) torna compreensível e justifica a técnica, assegurando que ela permita realizar as tarefas do tipo $T$, isto é, realizar o que é pretendido, sendo afirmações mais ou menos explícitas como proposições, definições e teoremas.

Para justificarmos a tecnologia, passamos a um nível superior de justificação - a teoria $\Theta$. A teoria é o discurso suficientemente amplo que serve para interpretar e justificar a tecnologia. Pode-se supor que exista a teoria da teoria, mas Chevallard (1999) considera suficiente a descrição de três níveis apresentados $(\tau, \theta, \Theta)$ para analisar uma tarefa.

Ressaltamos ainda que o relacionamento desta noção, de tipos de tarefas nos leva a refletir sobre técnicas, tecnologias e teorias associadas a gêneros de tarefas, ou seja, gêneros de técnicas, gêneros de tecnologia e gêneros de teorias.

Uma praxeologia matemática ou organização matemática é elaborada em torno de uma noção, ou conceito inerente à própria Matemática. Ou seja, refere-se à realidade Matemática que se pode construir em uma aula desta disciplina onde se estuda um determinado tema; devendo permitir que os estudantes atuem com eficácia para resolver problemas e, ao mesmo tempo, entender o que fazem de maneira racional. (BOSCH; CHEVALLARD, 2000).

\section{Modelo Epistemológico (ME)}

Um problema de investigação didática no âmbito da TAD se faz necessário questionar a forma de interpretar o Modelo Matemático (MM), o modelo epistemológico ${ }^{9}(\mathrm{ME})$, pois em diversos países, como mostra as pesquisas feitas por Dias (1993), Sierpinska et al. (2002),

\footnotetext{
9 Ao planejar sua aula, ou seja, na preparação do texto do saber, o professor de matemática elabora sua Organização Matemática e Didática com base em um Modelo Epistemológico, isto é, em uma maneira de compreender aquele saber.
} 
Wawro et al. (2011), Lindner (2003), Dorier (2002), Laugwitz (1974) e Harel (1990), que relatam as dificuldades que os alunos tem em enfrentar os tipos de tarefas presentes nos cursos de álgebra linear em seus respectivos países, logo o problema referente ao ensino e aprendizagem de álgebra linear não se restringe a um conjunto de habilidades e competências de um sujeito da instituição, mas pode se estar relacionado ao saber.

A dificuldade dos alunos em aprenderem AL, está inserido no âmbito da desarticulação entre esta disciplina e conteúdos já estudados pelos alunos, como função por exemplo. Nesta perspectiva, conforme assinala Bolea (2003), um importante aspecto a ser considerado no processo de Transposição Didática diz respeito ao âmbito do saber matemático.

Em geral um problema didático ${ }^{10}$ parte de um problema docente; e, na formulação de qualquer problema didático, o didata sempre utiliza uma descrição e uma interpretação que denomina ME.

O ME não é algo abstrato, em geral, pode estar vinculados e determina às práticas dos professores de matemática em uma dada instituição I, neste aspecto Gascón (2001), afirma que: "para começar a descrever e explicar a prática profissional do professor de matemática em sala de aula, pode nos colocar em diferentes perspectivas teóricas".

Segundo Gascón (1994) grande parte das pesquisas em Educação Matemática foca um domínio específico do saber matemática como álgebra elementar, por exemplo, onde o saber matemático é assumido pelas instituições, de tal forma que não há lugar para interpretações diferentes. Para o autor, este é um discurso que se põe como uma situação "normal" no sistema de ensino, onde o saber matemático não é questionado, o que torna pouco evidente e efetiva a distinção entre o saber sábio ${ }^{11}$ e o saber ensinado ${ }^{12}$. O saber sábio impõe-se como modelo de referência para o saber ensinado e é assumido muitas vezes sem as devidas adaptações para as instituições de ensino.

Segundo Boch e Gascón (2010), encontram-se ao longo do tempo várias formas da OM escolar no ensino, pois se apoiam de certa maneira em um ME, que está relacionado ao que se refere a ensinar e aprender matemática e uma instituição, que chamaremos de modelo didático. Este ME nos da compreensão da prática e das tarefas presentes nos livros didáticos, desenhando assim este ME.

Na medida em que os modelos didáticos permanecem implícitos, sem questionamento e na medida em que as formas de organizar os ensinamentos de matemática são apresentadas, sem qualquer justificativa explícita ou raciocínio além dos critérios genéricos, dizemos que este é um modelo didático espontâneo ${ }^{13}$, que é a prática espontânea do professor, como por exemplo, um professor resolve a uma equação polinomial do $2^{\circ} \mathrm{grau}$, geralmente utilizando a fórmula resolutiva de Bháskara e esta prática é comum entre a maioria dos professores no Brasil.

$\mathrm{Na}$ instituição IFPA, a respeito de um tipo de tarefas $\mathrm{T}$, com relação ao objeto $O$ Transformação Linear (TL) - existe em geral uma única técnica $\tau$, ou pelo menos um

\footnotetext{
10 Problema didático do professor, aqui com o tema Transformações Lineares, como o da construção de um sistema de tarefas traduzidos em organizações praxeológicas de complexidade crescente.

${ }^{11}$ É cultural, precisa ser difundido ensinado.

12 É o saber efetivamente ensinado pelo professor durante uma aula.

${ }^{13}$ As tarefas de ensino não estão organizadas de antemão em todos os seus detalhes, além do discurso teórico e tecnológico, por sua vez, ser pouco sistematizada e implícito (CHEVALLARD, BOSH e GASCÓN, 2001, p.3).
} 
pequeno número de técnicas $\left(\tau_{1}, \tau_{2}, \tau_{3}\right.$, etc.) institucionalmente reconhecidas, excluindo técnicas alternativas possíveis $\tau_{i}$, que podem existir efetivamente, mais em outras instituições $\left(I_{1}, I_{2}\right.$, etc.).

Neste artigo direcionamos nossa pesquisa nas tarefas e assim evidenciaremos o modelo epistemológico dominante (MED) do objeto O, Transformação Linear, por meio de discussão da organização matemática do livro didático dos autores Alfredo Steinbruch e Paulo Winterle (1987), obra intitulada Álgebra Linear, em termos do tipo de tarefas e gênero de tarefas, técnicas e justificações da razão de ser do objeto, na instituição IFPA.

\section{Definição de Transformação Linear e análise dos dois tipos de tarefas}

A Álgebra Linear tem aplicação em inúmeras áreas como computação gráfica, genética, redes elétricas entre outras. Trataremos de definir nosso objeto $O$, a Transformação Linear $(\mathrm{TL})$, no livro didático analisado, que define o objeto $O$ da seguinte forma:

Sejam $U$ e $\vee$ espaços vetoriais. Uma aplicação T: $U \rightarrow \vee$ é chamado transformação linear de $U$ em $V$ se, e somente se:

(a) $T(u+v)=T(u)+T(v), \forall u, v \in U$.

(b) T $\left(\alpha_{\mathrm{u}}\right)=\alpha \mathrm{T}(\mathrm{u}), \forall \alpha \in \mathrm{R}$ e $\forall \mathrm{u} \in \mathrm{U}$ (STEINBRUCH; WINTERLE, 1987, p. 152).

Após anunciarem a definição de $\mathrm{TL}$, os autores do livro começam apresentando as tarefas as quais analisamos a seguir. Começaremos pelo tipo de tarefa $T_{1}$ : Verificar se as transformações são lineares. Esse tipo de tarefa possuirá tarefas denotadas por $t_{i}\left(t_{1}, t_{2}, t_{3}\right.$, etc.).

A primeira tarefa, denotada por $t_{1}$, é assim descrita:

$t_{1}$ - Dada $T: R^{2} \rightarrow R^{3}$, onde $T(x, y)=(3 x,-2 y, x-y)$.Verifique se é linear: (STEINBRUCH; WINTERLE, 1987, p. 152).

Os autores recomendam a técnica $\tau$ para resolver a tarefa $t_{1}$, utilizando os elementos da definição de uma $T L$. Utilizando a técnica $\tau$ - definição de $T L$, necessitamos abstrair os vetores genéricos no $R^{2}$, ou seja, $u=\left(x_{1}, y_{1}\right)$ e $v=\left(x_{2}, y_{2}\right)$, então:

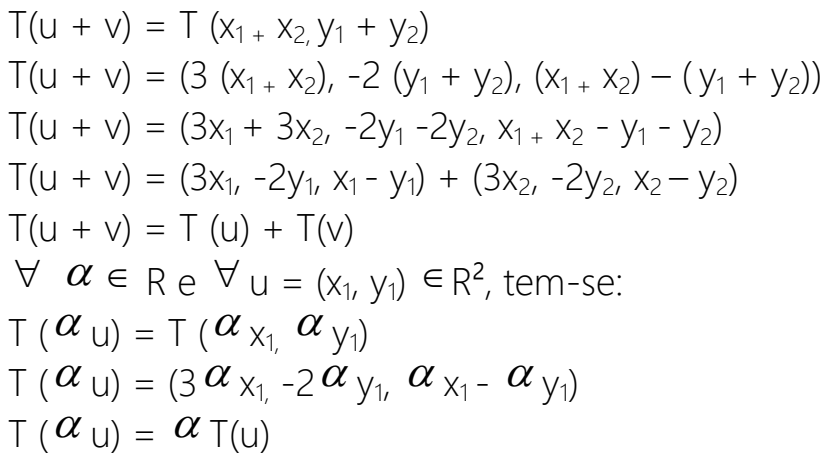

Note-se que na resolução da tarefa tı pela técnica $\tau$, não há articulação com a ideia de função, deixando o leitor confuso no que tange a ideia do que é transformar algo do $R^{2}$ - os vetores - $\left(x_{1}, y_{1}\right)$ e $\left(x_{2}, y_{2}\right)$ para $\circ R^{3}$ pares ordenados $\left(3 x_{1},-2 y_{1}, x_{1}-y_{1}\right)$ e $\left(3 x_{2},-2 y_{2}, x_{2}-y_{2}\right)$. Nenhuma representação gráfica é apresentada pelos autores na tarefa, que ajudaria no entendimento do que esta representando esta transformação.

Não há referência por parte do autor, que no estudo deste objeto não se trabalha com pares ordenados e sim com vetores, pois os alunos podem confundir com funções de duas 
ou três variáveis, pois os gráficos do sistema cartesiano parecem representar pontos no plano e no espaço, mas no caso das TL a relação é entre vetores.

A segunda tarefa $t_{i}$ que selecionamos é mais simples, porém importante na compreensão de uma TL: $t_{2}$ - Dada $T: R \rightarrow R$, onde T $(x)=3 x$. (STEINBRUCH; WINTERLE, 1987, p. 153).

Para saber se a transformação é linear os autores usam a técnica da tarefa anterior:

Sendo $u=x_{1}$ e $v=x_{2}$ vetores em $R$, então:

$T(u+v)=T\left(x_{1}+x_{2}\right)$

$T(u+v)=3\left(x_{1}+x_{2}\right)$

$T(u+v)=3 x_{1}+3 x_{2}$

$T(u+v)=T(u)+T(v)$

$\mathrm{T}\left(\alpha_{\mathrm{u}}\right)=\mathrm{T}\left(\alpha_{\mathrm{X}_{1}}\right)$

$\mathrm{T}\left(\alpha_{\mathrm{u}}\right)=3 \alpha_{\mathrm{X}_{1}}$

$\mathrm{T}\left(\alpha_{\mathrm{u}}\right)=\alpha_{\left(3 \mathrm{x}_{1}\right)}$

$\operatorname{Logo} \mathrm{T}\left(\alpha_{\mathrm{u}}\right)=\alpha \mathrm{T}(\mathrm{u})$

Os autores poderiam utilizar a geometria para auxiliar o aluno nesta tarefa, com a perspectiva de diminuir a dificuldade na percepção das relações entre geometria e álgebra e não tirar o sentido para o aprendizado dos alunos. A transformação nesta tarefa é um operador linear e a ideia é mostrar operadores do $R^{1} \rightarrow R^{1}, R^{2} \rightarrow R^{2}$, do $R^{3} \rightarrow R^{3}$ até generalizar para $T: R^{n} \rightarrow R^{n}$, isto é, para um $T(x)=r x$ ( $x$ é um vetor $R^{n}$ e $r$ um vetor em $R$, construindo deste modo tarefas em nível de complexidade crescente. A Figura 2 ilustra uma transformação $T(x)=2 x$. Esta tarefa poderia ser apresentada como primeira, ou seja deveria ser a $t_{1}$ por ter um nível de complexidade menor (Figura 2).

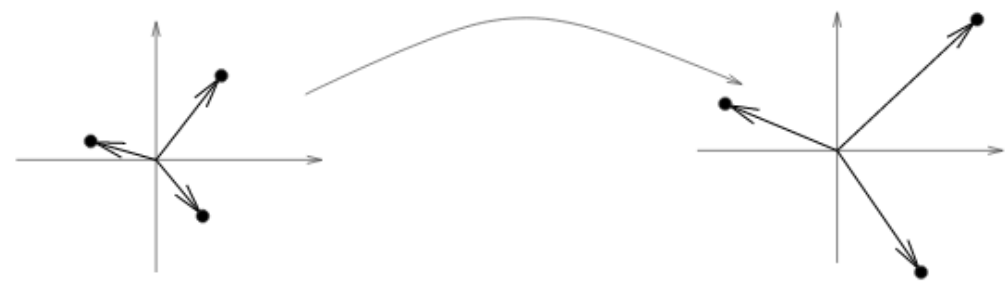

Figura 2 - Representação gráfica T $(x)=2 x$. Fonte: Figueiredo et al. (2012, p.19).

A terceira tarefa $t_{3}$ do tipo $T_{1}$ segue próxima da tarefa $t_{2}$, mas com complexidade maior: $t_{3}$ - Dada $T: R \rightarrow R$, onde $T(x)=3 x+1$ (STEINBRUCH; WINTERLE, 1987, p. 152). Os autores utilizam o recurso geométrico para mostrar que a Transformação Linear representa uma reta que passa pela origem, caso não passe não será linear.

Os autores utilizam como técnica, para resolver a $t_{3}$, a propriedade que chamaremos de $P_{1}$ : Em toda $T L T: V \rightarrow W$, a imagem do vetor $0 \in V$ é o vetor $0 \in W$, isto é: $T(0)=0$, a qual também decorre da definição, sendo que esta propriedade serve para provar que não é linear, mas se $\mathrm{T}(0)=0$, ainda assim teríamos que testar a definição. (STEINBRUCH; WINTERLE, 1987, p. 154).

Nesta tarefa os autores já apresentam um gráfico de uma função linear, pois já é um conhecimento que se supõe que os alunos já trazem consigo, para mostrar que esta não é uma $T L$, além de usar a propriedade da definição, isto é, ao substituir $u=x e v=y$ na transformação $T(u+v)=3(x+y)+1$, por conseguinte trabalhando com o $2^{\circ}$ membro da equação, temos: $3 x+3 y+1$. Nota-se que $T(u+v)=T(u)+T(v)$, não é verdade, pois teria 
que aparecer o número 2 no $2^{\circ}$ membro da equação. Logo a propriedade $1\left(P_{1}\right)$ é falha. Ainda nesta tarefa os autores não mostram a diferença para os leitores de $R$ como espaço vetorial euclidiano e $\mathrm{R}$ como conjunto dos números reais.

A quarta tarefa é descrita por: $t_{4}$ - Dada $T: R^{3} \rightarrow R^{2}$, onde $T(x, y)=(3 x+2,2 y-z)$ verifique se é linear (STEINBRUCH; WINTERLE, 1987, p. 155).

Notemos que em $t_{4}$ a terna vetorial $(x, y, z)$ será associado ao vetor $(x, y)$ pela $T L$, então $T(x, y)=(3 x+2,2 y-z)$, na qual $x=3 x+2$ e $y=2 y-z$

Nesta tarefa os autores utilizam como técnica a propriedade $P_{1}\left(\tau=P_{1}\right)$.

Utilizando vetores genéricos no $R^{2}$, tem-se $u=\left(x_{1}, y_{1}\right)$ e $v=\left(x_{2}, y_{2}\right)$, então pela $P_{1}$, temos: $T(0,0,0)=(3.0+2,2.0-0)$. Isso revela que $T(0,0,0)=(2,0) \neq(0,0)$, lê-se: $A$ transformação $T(0,0,0)$ que tem como imagem $(2,0)$ é diferente de $(0,0)$. Mostra-se que não pode ser linear não sendo necessário mostrar as outras duas propriedades da definição.

A quinta tarefa do tipo $T_{1}$, possui maior complexidade, exige detalhes algébricos mais refinados, ela está anunciada assim:

$t_{5}$ - Dada $T: R^{2} \rightarrow R^{3}$, onde $T(x, y)=\left(x^{2}\right.$, 3y) (STEINBRUCH; WINTERLE, 1987, p. 155).

A técnica apresentada no livro didático é descrita no quadro 1 , novamente, se da pelo uso dos elementos da definição, pois sendo $u=\left(x_{1}, y_{1}\right)$ e $v=\left(x_{2}, y_{2}\right)$, então: $T(u+v)=T\left(x_{1}+\right.$ $\left.\mathrm{x}_{2}, \mathrm{y}_{1}+\mathrm{y}_{2}\right)$. Então substituímos o domínio na variável para encontrarmos a imagem, como segue:

Desenvolvendo o primeiro membro da equação, temos:

$T(u+v)=\left(\left(x_{1}+x_{2}\right)^{2}, 3\left(y_{1}+y_{2}\right)\right)$ desenvolvendo o produto notável

$T(u+v)=\left(\left(x^{2}{ }_{1}+2 x_{1} x_{2}+x^{2}{ }_{2}, 3 y_{1}+3 y_{2}\right)\right)$ enquanto que se desenvolvermos o segundo membro, temos:

$T(u)+T(v)=\left(x^{2}{ }_{1}+x_{2}^{2}, 3 y_{1}+3 y_{2}\right)$ então conclui-se:

$T(u+v) \neq T(u)+T(v)$.

Mesmo usando como técnica $(\tau)$ a propriedade $P_{1}$, isto é, sendo uma $T L: V \rightarrow W$, onde $T(0)=0$ (vetor nulo), esta não é suficiente para mostrar que é uma $T L$, recorre-se então as propriedades da definição e verifica-se que a primeira é falha.

A sexta tarefa $t_{i}$ do tipo $T_{1}$ possui elementos axiomáticos da Álgebra Linear. A resolução dessa tarefa demanda um grau de abstração bem maior. Ela está assim anunciada: $t_{6}-\mathrm{A}$ transformação nula $T_{1}: V \rightarrow W$, onde $v \rightarrow 0$ ou $T(V)=0$ é linear, assim como simetria em relação a origem $T_{2}: R^{3} \rightarrow R^{3}, v \rightarrow-v$, é linear (STEINBRUCH; WINTERLE, 1987, p. 156).

A técnica utilizada continua sendo os elementos da definição:

i) $\quad T(u+v)=-(u+v)=-u-v=T(u)+T(v)$

ii) $\quad T(\alpha u)=-\alpha u=\alpha(-u)=\alpha T(u)$

De acordo com a TAD ao classificar o livro didático como representante da Instituição / e as transformações lineares como o objeto $O$, caracterizamos a relação institucional com o objeto, expressada por $\mathrm{R}_{\mathrm{l}}(\mathrm{O})$, como aquela que apresenta uma razoável valorização da geometria no tipo de tarefa $T_{1}$, mas que reflete uma deficiência de articulação com função e uso exclusivo da definição como justificativa.

Prosseguiremos nossa análise com o segundo tipo de tarefas $T_{i}, a T_{2}$. Nesse tipo de tarefa a propriedade seguinte é fundamental: Se $T: V \rightarrow W$ é uma $T L$, então $T\left(a_{1} V_{1}+a_{2} V_{2}\right)=a_{1}$ $T\left(v_{1}\right)+a_{2} T\left(v_{2}\right), \forall a_{1}, a_{2} \in R e \forall v_{1}, v_{2} \in V$. Por essa propriedade, mostra-se que a imagem de 
uma combinação linear de vetores ${ }^{14}$ é uma combinação linear das imagens desses vetores, com mesmos coeficientes, assim a TL fica definida quando se conhecem as imagens dos vetores de uma base ${ }^{15} \mathrm{de} \mathrm{V}$.

Quadro 1 é apresentado organização praxeológica, referente a ti.

\begin{tabular}{|c|c|c|c|c|c|}
\hline $\begin{array}{l}\text { Organização } \\
\text { praxeológica }\end{array}$ & $\begin{array}{c}\text { Gênero de } \\
\text { tipo de tarefa }\end{array}$ & Tarefa $(\mathrm{t})$ : & Técnica $(\tau)$ : & $\begin{array}{l}\text { Tecnologia } \\
(\theta):\end{array}$ & Teoria $(\Theta)$ : \\
\hline$t_{1}$ & Transformar $\mathrm{T}$ & $\begin{array}{l}t_{1} \text { verificar } \\
\text { se } T(x, y)= \\
(3 x,-2 y, x- \\
y) \text {. é linear }\end{array}$ & $\begin{array}{l}\text { elementos da } \\
\text { definição }\end{array}$ & definição & $\begin{array}{l}\text { Álgebra } \\
\text { Linear }\end{array}$ \\
\hline & Transformar $\mathrm{T}$ & $\begin{array}{l}\text { verificar se } \\
\mathrm{T}(\mathrm{x}, \mathrm{y})=3 \mathrm{x} \\
\text { é linear }\end{array}$ & $\begin{array}{l}\text { elementos da } \\
\text { definição }\end{array}$ & definição & $\begin{array}{l}\text { Álgebra } \\
\text { Linear }\end{array}$ \\
\hline$t_{3}$ & Transformar $\mathrm{T}$ & $\begin{array}{c}\text { verificar se } \\
T(x)=3 x+ \\
1 \text { é linear }\end{array}$ & $\begin{array}{c}\text { elementos da } \\
\text { definição, } \\
\text { elementos da } \\
\text { definição, } \\
\text { ideia de } \\
\text { função e a } \\
\text { propriedade } \\
1\end{array}$ & $\begin{array}{c}\text { definição } \\
\text { função linear }\end{array}$ & $\begin{array}{l}\text { Álgebra } \\
\text { Linear }\end{array}$ \\
\hline $\mathrm{t}_{4}$ & Transformar $\mathrm{T}$ & $\begin{array}{l}\text { verificar se } \\
T(x, y)=(3 x \\
+2,2 y-z) \\
\text { é linear }\end{array}$ & $\begin{array}{c}\text { Propriedade } \\
\mathrm{P}_{1}\end{array}$ & $\begin{array}{c}\text { Definição } \\
\text { Propriedade } \\
1\end{array}$ & $\begin{array}{l}\text { Álgebra } \\
\text { Linear }\end{array}$ \\
\hline$t_{5}$ & Transformar & $\begin{array}{l}\text { verificar se } \\
T(x, y)=\left(x^{2},\right. \\
\text { 3y) é linear }\end{array}$ & $\begin{array}{l}\text { elementos da } \\
\text { definição }\end{array}$ & $\begin{array}{c}\text { Definição } \\
\text { Propriedade } \\
1\end{array}$ & $\begin{array}{l}\text { Álgebra } \\
\text { Linear }\end{array}$ \\
\hline$t_{6}$ & Transformar & $\begin{array}{c}\text { verificar se } \\
T(v)=0 e \\
T(v)=-v \\
\text { são } \\
\text { lineares }\end{array}$ & $\begin{array}{l}\text { elementos da } \\
\text { definição }\end{array}$ & $\begin{array}{l}\text { Definiçãa de } \\
\text { TL } \\
\text { coordenadas } \\
\text { no } \mathrm{R}^{3}\end{array}$ & $\begin{array}{l}\text { Álgebra } \\
\text { Linear }\end{array}$ \\
\hline
\end{tabular}

\footnotetext{
${ }^{14}$ Uma combinação linear de um conjunto $S$ de vetores de um espaço vectorial $V$ sobre um corpo $K$ é uma soma finita $a_{1} v_{1}+\ldots+a_{n} v_{n}$ onde $v_{1}, \ldots . ., v_{n}, v \in S$ e $a_{1} v_{1}+\ldots+a_{n} v_{n}, a \in K$

15 Se É um espaço vectorial sobre um corpo K chama-se base de E a um conjunto de vectores de $\mathrm{E}$ linearmente independentes que gera $\mathrm{E}$.
} 
No Quadro 1 apresentamos a organização praxeológica referente aos tipos de tarefa $T_{1}$. As tarefas são apresentadas, inicialmente, sem um nível de complexidade crescente dos conteúdos, sendo tarefas bem pontuais, sem articulação com os conteúdos já vistos no ensino básico, como função e geometria analítica. No Quadro 1 as técnicas são justificadas pela definição de TL.

O leitor memoriza a técnica de resolução das tarefas, sem desenvolver as suas próprias estratégias de solução, chegando de maneira rápida à formalização, sem se questionar para que ser este objeto TL.

Analisaremos algumas tarefas $t_{i}$, do tipo de tarefa $T_{2}$, no qual os autores mostram como exemplo a $t_{1} \in T_{2}$, com o seguinte enunciado: Seja $T: R^{3} \rightarrow R^{2}$ uma $T L$ e $B=\left\{V_{1}, V_{2}, V_{3}\right\}$ uma base do $R^{3}$, sendo $v_{1}=(0,1,0), v_{2}=(1,0,1)$ e $v_{3}=(1,1,0)$. Determinar $T(5,3-2)$, sabendo que $T\left(v_{1}\right)=(1,-2), T\left(v_{2}\right)=(3,1)$ e $T\left(v_{3}\right)=(0,2)$ (STEINBRUCH; WINTERLE, 1987, p. 161).

Expressemos $v=(5,3-2)$ como combinação linear dos vetores da base $B$ :

$(5,3-2)=a_{1}(0,1,0)+a_{2}(1,0,1)+a_{3}(1,1,0)$

Resultando no sistema a seguir:

$\left\{\begin{array}{l}a_{2}+a_{3}=5 \\ a_{1}+a_{3}=3 \\ a_{1}=-2\end{array}\right.$

Após resolver o sistema linear se obtém $a_{1}=-4, a_{2}=-2, a_{3}=-2$.

Substituindo-se $a_{1}=-4, a_{2}=-2, a_{3}=-2$ na combinação linear:

$(5,3-2)=a_{1}(0,1,0)+a_{2}(1,0,1)+a_{3}(1,1,0)$, teremos:

$(5,3,-2)=-4 v_{1}-2 v_{2}+7 v_{3}$

Aplicando a transformação - $T$ em todos os termos, tem-se:

$T(5,3,-2)=-4 T\left(v_{1}\right)-2 T\left(v_{2}\right)+7 T\left(v_{3}\right)$

Substituindo as imagens de $T\left(v_{1}\right), T\left(v_{2}\right)$ e $T\left(v_{3}\right)$, obtém-se:

$T(5,3,-2)=-4(1,-2)-2(3,1)+7(0,2)$

$T(5,3,-2)=(-10,20)$

$\mathrm{Na}$ tarefa $\mathrm{t}_{1}$ proposta os autores não deixam claro para o leitor que esta sendo utilizado como ferramenta a noção de domínio e imagem de função, ou seja, não esta articulando a técnica com que o aluno do Ensino Superior tem de conhecimento prévio que é a prática de cálculo com tipos de funções.

A segunda tarefa do tipo $T_{2}$ é a tarefa $t_{2}$, que se enuncia:

Seja $T: R^{3} \rightarrow R^{2}$, onde $T(x, y, z)=(x+2 y+2 z, x+2 y-z,-x+y+4 z)$. Determinar:

$t_{21}: u \in R^{3} / T(u)=(-1,8,-11)$ ? e;

$t_{22}: v \in R^{3} / T(v)=v(S T E I N B R U C H ;$ WINTERLE, 1987, p. 165).

Os autores resolvem a subtarefa $t_{21}$ igualando $T(u)=(-1,8,-11)$ a $T(x, y, z)=(x+2 y+2 z$, $x+2 y-z,-x+y+4 z)$ e então surge um sistema linear de três incógnitas e três equações onde encontraremos os valores do vetor $u$. Desse modo, pela igualdade, $(x+2 y+2 z, x+2 y-z,-$ $x+y+4 z)=(-1,8,-11)$, obtemos o sistema a seguir

$$
\left\{\begin{array}{l}
x+2 y+2 z=-1 \\
x+2 y-z=8 \\
-x+y+4 z=-11
\end{array}\right.
$$

Resolvendo esse sistema, encontramos $u=(1,2,-3)$.

Na subtarefa $t_{22}$ como não foi dado $T(v)$ então os autores usam um genérico $T(v)=(x$, $y, z)$ e por sistemas lineares determina a solução do sistema indeterminado da forma $v=$ 
$(2 z,-z, z)$. Apesar de não ter que determinar $T(u)$ e sim "u" está no gênero de tipo de tarefa $T_{2}$, pois se trata de determinar o domínio da transformação $T$, no caso de determinarmos $u$ e a imagem desta transformação no caso de determinarmos $T(u)$.

Pensando em um vetor qualquer para $v=(x, y, z) \in V^{3}$, tem-se:

$(x+2 y+2 z, x+2 y-z,-x+y+4 z)=(x, y, z)$

$x+2 y+2 z=x$

$x+2 y-z=y$

$-x+y+4 z=z$

Então: $v=(2 z,-z, z)=z(2,-1,1), \forall z \in V^{3}$.

Ainda com relação ao tipo de tarefa $T_{2}$, os autores Steinbruch e Winterle (1987, p. 165) prescreve a seguinte tarefa, que chamaremos de $t_{3}$ : Dada $T: R^{2} \rightarrow R^{3}$, uma $T L$ e que $T(1,-1)$ $=(3,2,-2)$ e $T(-1,2)=(1,-1,3)$, determinar $T(x, y)$.

Os autores iniciam que $\{(1,-1),(-1,2)\}$ é uma base do $R^{3}$ e aplicando a propriedade $P_{2}$, "Se T: $V \rightarrow$ W é uma TL, então $T\left(a_{1} v_{1}+a_{2} v_{2}\right)=a_{1} T\left(v_{1}\right)+a_{2} T\left(v_{2}\right)$ para $\forall a_{1}, a_{2} \in R$ e $\forall v_{1}, v_{2} \in$ $V^{\prime \prime}$, obtém-se: $(x, y)=a(1,-1)+b(-1,2)$. Dessa igualdade, surge um sistema linear e ao resolvermos temos como resposta $a=2 x+y e b=x+y$. Portanto, $T(x, y)=a T(1,-1)+b T(-1$, 2). Onde $a=2 x+y$ e $b=x+y$. Substituindo as imagens de $T(1,-1)=(3,2,-2)$ e $T(-1,2)=$ $(1,-1,3)$ temos:

$$
\begin{aligned}
& T(x, y)=(2 x+y)(3,2,-2)+(x+y)(1,-1,3) \rightarrow \\
& (2 x \cdot 3+2 x \cdot 1+y \cdot 3+y \cdot 1)=(6 x+2 x+3 y+y)=(7 x+4 y) \\
& (2 x \cdot 2+x \cdot(-1)+y \cdot 2+y \cdot(-1))=(4 x-x+2 y-y)=(3 x+y) \\
& (2 x \cdot(-2)+x \cdot 3+y \cdot(-2)+y \cdot 3)=(-4 x+3 x-2 y+3 y)=(-x+y) \\
& T(x, y)=(7 x+4 y, 3 x+y,-x+y) .
\end{aligned}
$$

Em Steinbruch e Winterle (1987, p. 167) é apresentada a tarefa $t_{4}$ : Sabendo que T: $R^{2} \rightarrow$ $R^{2}$ é uma $T L$, em especial um Operador Linear - $O L$, e que $T(1,0)=(3,-2)$ e $T(0,1)=(1,4)$, Determinar $T(x, y)$.

A técnica $\tau$ que os autores recorrem é a premissa que qualquer vetor no $R^{2}$ pode ser escrito como uma combinação linear como a base canônica: $(1,0)$ e $(0,1)$. Esse recurso permite estabelecer que $(x, y)=x(1,0)+y(0,1)$, em seguida aplica a Transformação $T$ : $T(x, y)$ $=x T(1,0)+y T(0,1)$. Substituindo os valores das imagens de $(1,0)$ e $(0,1)$ dadas no enunciado de $_{4}$, temos $T(x, y)=x(3,-2)+y(1,4)$. Determinando assim o valor de $T(x, y)=(3 x+y,-2 x+4 y)$.

$A$ tarefa $t_{4}$ é semelhante à tarefa $t_{3}$, mas a complexidade é menor. Os autores em nenhum momento relatam que estão usando a ideia de domínio e imagem de função, pois substituem direto na combinação linear construída a partir da base canônica. No quadro 2 é possivel se verificar a organização praxeológica, referente ao tipo de tarefas $T_{2}$.

Todas as tarefas $t_{i}$ pertencentes aos tipos $T_{1}$ e $T_{2}$ revelam as técnicas $\tau$ predominantes no livro didático de Steinbruch e Winterle (1987).

A razão de ser para se ensinar as Transformações Lineares é a aplicação da técnica, pois os alunos devem se perguntar ao lerem as propostas das tarefas enunciadas pelos autores, para que e porque se estudar tal temática? Algumas tarefas são resolvidas com os conteúdos vistos no ensino médio, como função como tecnologia, não deixando claro ao leitor que é uma transformação de um espaço vetorial em outro.

Do ponto de vista de uma organização praxeológica, apenas usar elementos da definição como técnica, deve ser levado em conta, para que na análise destas técnicas utilizadas pelos alunos, seja possível compreender melhor a razão de algumas dificuldades a serem superadas. 
Quadro 2: Organização praxeológica, referente a $t_{\text {i }}$.

\begin{tabular}{|c|c|c|c|c|c|}
\hline $\begin{array}{l}\text { Organizaç } \\
\text { ão } \\
\text { praxeológ } \\
\text { ica }\end{array}$ & $\begin{array}{l}\text { Gênero de } \\
\text { tipo de } \\
\text { tarefa }\end{array}$ & Tarefa $(\mathrm{t})$ : & Técnica $(\tau)$ : & $\begin{array}{c}\text { Tecnologia } \\
(\theta):\end{array}$ & $\begin{array}{c}\text { Teoria } \\
(\Theta) \text { : }\end{array}$ \\
\hline$t_{1}$ & $\begin{array}{l}\text { Determinar } \\
\mathrm{T}(\mathrm{x}, \mathrm{y}, \mathrm{z})\end{array}$ & $\begin{array}{c}\text { Determinar a } T \\
(5,3-2) \text { dado a } \\
\text { base B }\end{array}$ & $\begin{array}{l}\text { Combinação } \\
\text { linear, resolução } \\
\text { de sistemas } \\
\text { lineares, } \\
\text { domínio e } \\
\text { imagem }\end{array}$ & $\begin{array}{c}\text { Sistemas } \\
\text { lineares } \\
\text { Função }\end{array}$ & $\begin{array}{l}\text { Álgebra } \\
\text { Linear }\end{array}$ \\
\hline$t_{2}$ & $\begin{array}{c}\text { Determinar } \\
\text { a } \\
\text { transformaç } \\
\text { ão T }\end{array}$ & $\begin{array}{c}\text { Aqui as } \\
\text { subtarefas: } \\
\mathrm{t}_{21:} \mathrm{u} \in \mathrm{R}^{3} / \mathrm{T}(\mathrm{u}) \\
=(-1,8,-11) \mathrm{e} \\
\begin{array}{c}\mathrm{t}_{22}: \mathrm{v} \in \mathrm{R}^{3} / T(\mathrm{v}) \\
=\mathrm{v} .\end{array}\end{array}$ & $\begin{array}{l}\text { Conceito de } \\
\text { função, em } \\
\text { especial domínio } \\
\text { e imagem } \\
\text { Resolução de } \\
\text { um sistema } \\
\text { linear }\end{array}$ & $\begin{array}{c}\text { Sistemas } \\
\text { lineares } \\
\text { Função }\end{array}$ & $\begin{array}{l}\text { Álgebra } \\
\text { Linear }\end{array}$ \\
\hline$t_{3}$ & $\begin{array}{c}\text { Determinar } \\
\text { a } \\
\text { transformaç } \\
\text { ão T }\end{array}$ & $\begin{array}{c}\text { Determinar a } \\
\text { transformação } \\
T(x, y)\end{array}$ & $\begin{array}{l}\text { Conceito de } \\
\text { função, em } \\
\text { especial domínio } \\
\text { e imagem } \\
\text { Resolução de } \\
\text { um sistema } \\
\text { linear } \\
\text { Combinação } \\
\text { linear } \\
\text { Utilização da } \mathrm{P}_{2}\end{array}$ & $\begin{array}{c}\text { Sistemas } \\
\text { lineares } \\
\text { Função } \\
\mathrm{P}_{2}\end{array}$ & $\begin{array}{l}\text { Álgebra } \\
\text { Linear }\end{array}$ \\
\hline $\mathrm{t}_{4}$ & $\begin{array}{c}\text { Determinar } \\
\text { a } \\
\text { transformaç } \\
\text { ão T }\end{array}$ & $\begin{array}{c}\text { Determinar a } \\
\text { transformação } \\
T(x, y)= \\
(3 x+y, \\
2 x+4 y) .\end{array}$ & $\begin{array}{c}\text { Ideia de } \\
\text { Combinação } \\
\text { linear } \\
\text { Ideia de domínio } \\
\text { e imagem }\end{array}$ & $\begin{array}{c}\text { Combinação } \\
\text { linear } \\
\text { Função }\end{array}$ & $\begin{array}{l}\text { Álgebra } \\
\text { Linear }\end{array}$ \\
\hline
\end{tabular}

A algoritmização é uma maneira de diminuir a aparência de novidade, evitando a dificuldade conceitual, isto é, pensamos que seja uma maneira de driblar ainda mais as dificuldades do objeto transformação linear - TL. Quando os professores fazem a transposição didática interna, ou seja, ao passar o conteúdo em sala em uma versão didática, os algoritmos diversas vezes aparecem, que nem sempre são justificados pelo professor, muitas vezes por não compreender a razão de ser do objeto.

Evidenciamos que a OM do livro analisado não apresenta articulações e integrações de praxeologias que favoreçam um fazer mínimo racional sobre os objetos sistemas lineares e espaços vetoriais, pois a tecnologia, que justifica a técnica apresentada é a utilização direta da definição de espaços vetoriais.

A OMD que analisamos na obra principal de ensino de AL no IFPA, segundo nosso olhar, apresentam tarefas pontuais, e portanto atomizadas, conforme (FONSECA, 2004; 
BOSCH; FONSECA; GASCÓN, 2004). As condições que o livro coloca ao nosso ver são que os sistemas lineares são movimentados em separado, não há tarefas que evocam o alcance da técnica, isto é, não há articulação a partir de novas tarefes até chegar em TL.

Os tipos de tarefa são apresentadas a partir de aplicação direta das definições. O livro utiliza-se do formalismo da AL para desenvolver sua OMD e acaba se transformando em obstáculo no processo de ensino desta disciplina de curso superior, assim como pensa (DORIER, 1995; DORIER, 1997).

Tal formalismo deixa de lado comentários importantes sobre os temas estudados, como, por exemplo a articulação entre os sistemas lineares, incluindo os homogêneos com os estudos das $\mathrm{TL}$, portanto um estudo tecnicista, com mera aplicação da técnica apresentada na definição.

Os autores resolvem um exemplo e propõe aos alunos algo semelhante. Não consideramos as $t_{i}$, efetivamente um problema, pois as tarefas e as técnicas utilizadas não permitem o pensar dos alunos sobre os objetos. São inteligíveis e confiáveis dada as condições de seu emprego, tendo sua importância satisfatória, para que se propõe.

\section{Considerações Finais}

No livro de álgebra linear analisado, as tarefas são apresentadas sem um nível de complexidade crescente dos conteúdos, portanto são tarefas pontuais, ou seja, de não haver uma justificativa tecnológica e nem articulação explícita com os conteúdos já vistos no ensino básico, como função e geometria analítica, com informações prontas, não exigindo muita interpretação.

Os sistemas de tarefas apresentadas na obra analisada e por conseguinte a prática do professor que tem como lente apenas esta obra leva os estudantes a memorizar algoritmos sem compreender as propriedades e a lógica envolvida na técnica visada, pois memorizam os processos de resolução das tarefas dadas como tarefas, sem desenvolver as suas próprias estratégias de solução, chegando de maneira rápida à formalização.

O discurso teórico-tecnológico que esclarece as técnicas, no livro didático, apareceu de forma aleatória sem ser abordado explicitamente ou com uma justificativa evidente das técnicas utilizadas.

Conforme às análises, os livros dão suporte ao professor e os alunos, mas que de um modo geral não são capazes de entender o que é transformar espaços vetoriais em espaços vetoriais, pois às tarefas são apresentadas como um processo algorítmico, como uma estratégia de diminuir a dificuldade na disciplina.

As tarefas evocadas pelos autores do livro são meras aplicação das definições apresentadas, pois nas tarefas analisadas constatamos tarefas: verificar se $T$ é uma transformação linear e determinar a transformação $T(x, y)$. Logo, a atividade matemática dos alunos se dar em coerente com os conteúdos contidos nos livros. Destacamos, também, uma consequência do fenômeno da atomização das OM contidas no livro analisado é a escassez presença da atividade de modelagem matemática na instituição de ensino superior em questão, o IFPA, no que diz respeito, já que as atividades perpassam pela aplicação direta da definição apresentada, pois nesses manuais caberia tratar as transformações lineares a partir de tarefas com sistemas lineares.

A Teoria Antropológica do Didático nos encaminha que este tipo de OM presente no livro analisado, se torna uma restrição para se implementar um processo onde o aluno 
possa modelar, já que a forma de interpretar as OM propostas na obra, é uma outra restrição que resulta de algo, além da estrutura das praxeologias matemáticas escolares tais como: a forma de interpretar a atividade matemática por parte das instituições escolares, o que LUCAS et al. (2014) denominaram de modelo epistemológico dominante.

As possibilidades da organização praxeológica nesses dois tipos de tarefas $T_{1}$ e $T_{2}$, não ampliam as relações das técnicas de resolução das tarefas apresentadas, associadas ao discurso teórico-tecnológico. Nos dois tipos de tarefa discutidos $T_{1}$ e $T_{2}$ é possível dar indícios que o modelo epistemológico dominante presente no livro analisado é utilizar a definição de $T L$, respondendo ao objetivo do trabalho.

A razão de ser para se ensinar as Transformações Lineares na obra se dá em aplicar a técnica, pois os alunos devem se perguntar ao lerem as propostas das tarefas enunciadas pelos autores, para que e porque se estudar tal temática? Algumas tarefas são resolvidas com os conteúdos vistos no ensino médio, como função como tecnologia, não deixando claro ao leitor que é uma transformação de um espaço vetorial em outro, pois tais praxeologias resumem-se na existência de uma técnica privilegiada associada a uma dada tarefa, isto é, há diversas tarefas associas a uma única técnica.

\section{Referências}

ANDRADE, R. C. D. A noção de tarefa fundamental como dispositivo didático para um percurso de formação de professores: o caso da geometria analítica. Tese (Doutorado). Universidade federal do Pará. 2012.

ANDRADE, R. C. D., GERRA, R. B. Tarefa fundamental em um percurso de estudo e pesquisa: um caso de estudo para o ensino da Geometria Analítica. Educação Matemática Pesquisa. n. 16. v. 4. 2014.

BOSCH, M.; FONSECA, C.; GASCÓN, J. Incompletitud de las organizaciones matemáticas locales en las instituciones escolares. Recherches en Didactique des Mathématiques, Grenoble: Sauvage, v. 24, n. 2-3, p. 205-250, $4^{0}$ trimestre. 2004.

BOSH, M; GASCÓN, J. Fundamentación antropológica de lãs organizaciones didácticas de los "talleres de prácticas matemáticas" a los "recorridos de estúdio e investigación. $4^{\circ}$ Congreso Internacional sobre la Teoría Antropológica de lo Didáctico. 2010.

BOLEA, P. C. El proceso de algebrización de organizaciones matemáticas escolares. Monografía del Seminario Matemático García de Galdeano, 29. Departamento de Matemáticas. Universidad de Zaragoza, 2003.

CARVALHO, J. B. P; LIMA, P. F. O uso da coleção didático de matemática. v.17, p.137-169. Brasilia, 2010.

CHEVALLARD, Y. Concepts fondamentaux de la didactique: perspectives apportées par um approche anthropologique. In: Recherchesen Didactique dês Mathématiques. v. 12 n01, p.73112, 1992.

CHEVALLARD, Y. Conceitos fundamentais da didactia: as perspectivas trazidas por uma abordagem antropológica. In: BRUN, Jean. Didáctica das Matemáticas. Lisboa: Instituto Piaget. Horizontes Pedagógicos, 1996, p.115-153. 
CHEVALLARD, Y. L'analise des pratiques enseignantes em théorie antropologique du didactique. Recherches em Didactique des Mathématiques. Grenoble La Pensée Sauvage, v.19, n.2, pp.221-265. 1999.

CHEVALLARD, Y, BOSCH, M., GASCÓN, J. Estudar matemáticas: o elo perdido entre o ensino e a aprendizagem. Tradução: Daisy Vaz de Moraes. Porto Alegre: Artmed Editora, 2001.

DIAS, M. A. Contribution à analyse d'un enseignement expérimental d'álgèbre linéaire en DEUG A première année. Mémoire de DEA. Paris: Université de Paris 7, 1993.

DORIER, J. L.; ROBERT, A; ROBINET, J.; ROGALSHI, M. The teaching of linear algebra in first year of French science university, in the Proceedings of the 18th conference of the international group for the Psychology of Mathematics Education, Lisbonne, vol. 4, p. 137144, 1994.

DORIER, J.L. L'enseignement de l'algébre linéaire en question. França: La Pensé Sauvage éditions. 1997. p. 291-297.

DORIER, J.L. Teaching Linear Algebra at University. In Tatsien Li (Ed.), Proceedings of the International Congress of Mathematicians, ICM (Vol. III, pp. 875-884). Beijing, China: Higher Education Press. 2002.

FIGUEIREDO, L.M.; CUNHA, M.O.; DEDOYA. H; CAMELIER, R. Álgebra II. Consorcio CEDERJ. Ministério da educação - MEC. 2014. Disponível em: < http://www2.ufpa.br/quimdist/livros_bloco_4/ALGEBRA\%20LINEAR/volume\%202/algLin2.pdf >. Acesso em: 29 jun. 2014.

FONSECA, C. Discontinuidades matemáticas y didácticas entre la Secundaria y la Universidad. 2004. 263 p. Tesis (Doctoral en Ciencias Matemáticas) - Departamento de Matemática Aplicada I, Universidad de Vigo, Vigo, 2004.

GASCON, J. Un nouveau modèle de l'algèbre élémentaire comme alternative à l'algèbre généralisé. Petit x, n 37, 43-63, 1994.

LUCAS, C., BON, C. F., GASCÓN, J., CASAS, J. O Fenômeno Didático Institucional da Rigidez e a Atomização das Organizações Matemáticas Escolares. Boletim de Educação Matemática, v. 28, n. 50, 2014.

HAREL, G. Using geometric models and vector arithmetic to teach high-school students basic motions in linear algebra. International Journal Mathematics Education, Science and Technology. vol. 21, n 3, pp. 387- 392, 1990.

LAUGWITZ, D. Motivation and Linear Algebra. Educational Stuidies in Mathematics 5. Holland: Dordrecht, 1974. p. 243-254.

LINDNER, W. CAS-Supported Multiple Representations in Elementary Linear Algebra. The Case of the Gaussian Algorithm. ZDM - The International Journal on mathematics education, v. 35, n. 2, p. 36-42, 2003.

MENSSOURI, D. Essai de délimitation em termes de problématiques dês effets de contratet de transposition: lês cãs de relations entre droites et équations dans les classes de Seconde et Première. Thèse. Grenoble: Université Joseph Fourier. 1994. 
SABO, R. D. Análise de livros didáticos do ensino médio um estudo dos conteúdos referentes a combinatória. Monografia. Curso de Pós-Graduação Centro universitário Fundação Santo André. São Paulo. 2007.

SIERPINSKA, A.; TRGALOVA, J.; HILLEL, J.; DREYFUS, T. Teaching and Learning Linear Algebra with Cabri. Research Forum paper, in The Proceedings of PME 23, Haifa University, Israel: 1999, v. 1, p. 119-134.

STEINBRUCH, A.; WINTERLE, P. Álgebra Linear. São Paulo: Editora Pearson, $4^{a}$ ed. 1987.

TELLES, R. A. de M. A Aritmética e a álgebra na matemática escolar. Educação Matemática em Revista. São Paulo: SBEM, ano 11, n. 16, p. 8 -15, 2004.

WAWRO, M; SWEENEY, G. F.; RABIN, J. M. Subspace in linear algebra: investigating students concept images and interactions with the formal definition. ZDM - The International Journal on mathematics education. v. 78, p. 1-19, 2011. 